Mediterránea Ser. Biol. (1985), n. ${ }^{\circ}$ 8. Págs. 103-123

\title{
EL CICLO DEL POTASIO EN DEHESAS DE QUERCUS ROTUNDIFOLIA Y QUERCUS PYRENAICA.
}

\author{
por \\ A. Escudero Berian 1, B. García Criado 2 y H. Alonso Peloche 1
}

\section{RESUMEN}

Hemos estudiado el ciclo del potasio en dehesas de Quercus rotundifolia Lam. y Quercus pyrenaica Willd. de la provincia de Salamanca.

El potasio es el elemento que se transfiere en mayores proporciones a través de frutos y de material herbáceo, que son materias altamente digestibles. Por ello, su transferencia al subsistema herbívoro es mayor que para los restantes nutrientes.

El potasio es también el macronutriente más intensamente extraído de los tejidos vegetables por el agua de lluvia.

Todo ello hace al potasio muy móvil y con intensas tasas de circulación a través del ecosistema.

\section{THE POTASSIUM CYCLE IN QUERCUS ROTUNDIFOLIA AND QUERCUS PYRENAICA.DEHESAS ·}

\section{SUMMARY}

A study was made of the potassium cycle in Quercus rotundifolia Lam. and Quercus pyrenai$c a$ Willd. dehesa ecosystems in the province of Salamanca, Spain.

Potassium was seen to be the element which was transferred in the greatest proportions through the fruits and herbaceous materials: these are highly digestible. Accordingly, the transference to the herbivore subsystem is greater than that of the remaining nutrients.

(1) Dpto. de Ecología, Facultad de Biología, Universidad de Salamanca.

(2) Centro de Edafología y Biología Aplicada de Salamanca. 
Potassium was also seen to be the macroelement which was most intensely leached from the plant tissues by rain water.

These aspects mean that potassium is extremely mobile and presents pronounced circulation rates through the ecosystem:

\section{INTRODUCCION}

Las dehesas constituyen el paisaje predominante en la provincia de Salamanca. Ocupan en ella una superficie de más de 500,000 ha, que suponen el $44 \%$ del territorio provincial. Representan un estado de equilibrio entre explotación y conservación que las hace especialmente interesantes desde el punto de vista científico.

Hace ya algunos años comenzamos a abordar por primera vez ciertos aspectos de los ciclos biogeoquímicos en estos ambientes (ver, por ejemplo, GOMEZ GUTIERREZ y col., 1980), que hemos ido gradualmente completando. Presentamos aquí un balance global de los ciclos minerales, con especial énfasis en el potasio en razón de su peculiaridad.

El potasio es uno de los elementos fundamentales para los vegetales. Figura entre los más abundantes, siendo generalmente superado tan solo por nitrógeno y calcio. No es preciso subrayar su papel en gran número de procesos vegetales básicos, como la fotosíntesis o la síntesis de aminoácidos, así como su carácter limitante en muchos suelos. En el caso particular de los suelos de dehesa de la provincia de Salamanca, este carácter limitante del potasio no suele manifestarse tan frecuentemente, porque aunque sus niveles rara vez llegan a ser los adecuados, suelen serlo bastante más que los de nitrógeno y fósforo (GARCIA RODRIGUEZ, 1964). Sin embargo, estos ecosistemas seminaturales se hallan sometidos a una explotación humana que conlleva una extracción de recursos. La exportación de nutrientes resultante muy raramente va acompañada de la necesaria restitución posterior en forma de fertilizantes, pues la escasa productividad de las dehesas hace prohibitivo el uso de estos últimos. Por ello, el mantenimiento de la producción vegetal en estos ecosistemas depende enteramente de la eficacia de sus comunidades en el reciclado de los nutrientes. Como se pondrá de manifiesto en este trabajo, el ciclo del potasio reviste unas características muy especiales que lo hacen quizá más susceptible que el de ningún otro elemento a alteraciones debidas a la acción humana. Por otra parte, presenta un interés científico indudable por su complejidad. 


\section{MATERIAL Y METODOS}

Para elaborar este trabajo, hemos seleccionado tres dehesas típicas situadas en la provincia de Salamanca. Dos de ellas se hallan pobladas por encinas; la tercera, por robles (Quercus pyrenaica Willd.). Naturalmente, la más extensa área de distribución de la encina nos ha obligado a prestar mayor atención a esta especie. Las poblaciones de encina son denominadas, respectivamente, Campillo y Servández; la de roble, Cilloruelo.

Algunas características de estas comunidades vegetales son las siguientes: la densidad de arbolado oscila, según las parcelas, entre 23 y $39 \mathrm{pies} / \mathrm{ha}$; la superficie del suelo cubierta por la proyección de las copas de los árboles supone entre 11.3 y $13.2 \%$ del total. La superficie restante se halla cubierta por un pastizal dominado por las gramíneas Agrostis castellana, Bromus madritensis o Elymus caputmedusae. Bajo las copas de los árboles, las gramíneas predominantes son Dactylis glomerata y Bromus mollis. Las características edáficas de las tres parcelas vienen resumidas en la tabla $\mathrm{I}$. La precipitación anual media oscila en torno a $500 \mathrm{~mm}$ para el área de Quercus rotundifolia y se acerca a $600 \mathrm{~mm}$ para la de $Q$. pyrenaica. La temperatura media anual se mueve entre 11.5 y $12.5^{\circ} \mathrm{C}$.

La producción de restos vegetales se estimó mediante recogida continua de los mismos (MEDWECKA-KORNAS, 1971) en recipientes adecuados, que fueron dispuestos en torno a árboles aislados de forma ordenada a lo largo de lî́neas radiales tomando como centro el tronco del árbol. De esta manerà pudimos determinar la variación de los aportes con la distancia al centro de cada árbol. La duración total del período de control ha sido de tres años.

Una disposición similar se adoptó para una serie de pluviómetros destinados a medir y recoger la «trascolación» o agua de lluvia que atraviesa la copa (throughfall, en la terminología inglesa). Un dispositivo en torno al tronco permitió recoger el agua de escorrentía por la superficie de ramas y troncos (stemflow).

Se tomaron también periódicamente muestras de material herbáceo para determinar la producción y extracción de bioelementos del paștizal.

Las determinaciones de potasio en el material vegetal se efectuaron.por calcinación y posterior disolución de las cenizas en una mezcla 
de $\mathrm{ClH} / \mathrm{NO}_{3} \mathrm{H} / \mathrm{H}_{2} \mathrm{O}$ en proporción respectiva de 1:1:8. Las muestras de agua se evaporaron previamente hasta sequedad; el residuo obtenido fue calcinado, y las cenizas, disueltas en la forma descrita.

De las disoluciones obtenidas se tomaron alícuotas para la determinación de potasio por espectrofotometría de absorción atómica previa adición de cesio para eliminar interferencias causadas por ionización. Otros elementos determinados en estos materiales fueron nitrógeno, fósforo, calcio, magnesio, sodio, hierro, cobre y manganeso.

\section{RESULTADOS Y DISCUSION}

\section{A. Incorporación y transferencias de potasio en la materia orgánica.}

El aislamiento de los pies de encina y roble de la dehesa determina una considerable heterogeneidad en la intensidad de circulación de nutrientes. Así, mientras que las cantidades de potasio en los restos orgánicos depositados anualmente bajo las copas de las encinas se mueven entre 9.8 y $20.4 \mathrm{~kg} / \mathrm{ha}$, en la zona situada inmediatamente por fuera del borde de la proyección de la copa estas aportaciones se reducen a cifras comprendidas entre 1.9 y 3.3 . $\mathrm{kg} / \mathrm{ha}$. Para la parcela de roble, la restitución de potasio al suelo bajo las copas supone entre el 5.8 y $10.1 \mathrm{~kg} / \mathrm{ha}$, mientras que en el contorno exterior al borde de la copa se reciben en torno a $1.7 \mathrm{~kg}$. Naturalmente, a medida que nos alejamos del árbol, la adición de potasio va disminuyendo. A $5 \mathrm{~m}$ del borde sólo se alcanzan en torno a $0.74 \mathrm{~kg} / \mathrm{ha}$ de potasio en los encinares y 0.69 en el robledal. A $9 \mathrm{~m}$ dẹl borde (distancia máxima a la que se suele observar llegada de materiales del árbol arrastrados por el viento), el acceso de potasio al suelo se reduce a $0.23 \mathrm{~kg} / \mathrm{ha}$ para las encinas y a 0.33 para los robles, que al poseer hojas de mayor tamaño y más susceptibles de ser arrastradas por el viento aporta comparativamente más que la encina a estas distancias más elevadas. Esta mayor sustracción de materiales hace así aparecer menores las cifras de restitución de nutrientes bajo la copa de los robles.

Numerosos investigadores han estudiado los ciclos de nutrientes en diversos ecosistemas forestales. A efectos de comparación con nuestros propios datos, hemos efectuado una grosera clasificación de los datos bibliográficos (BARES y WALI, 1979; COLE et al., 1978; DUVIGNEAUD, 1968; RAPP, 1967, 1969, entre otros muchos), repartiéndolos en dos grupos según que representen a ecosistemas aparentemente eutróficos y gozando en consecuencia de relativamente intensos flujos de nutrientes, o a ecosistemas más pobres. No es de extrañar, sin em- 
bargo, que incluso dentro de cada uno de estos grupos de ecosistemas, los rangos de variación de los parámetros del ciclo sean considerablemente amplios. Para ecosistemas «ricos» encontramos unos límites de variación en las adiciones anuales de potasio de 5 y $67 \mathrm{~kg} \mathrm{ha-1}$ año-1, mientras que en los más bien «pobres», estos límites se reducen a 2.7-12, con considerable solapamiento, pues, entre los dos grupos de ecosistemas. Por consiguiente, la dehesa se encuentra en una posición intermedia en lo que respecta a la restitución anual de potasio en la zona directamente cubierta por las copas de los árboles.

Otro aspecto a tener en cuenta es la forma en que se efectúan los retornos de nutrientes. Los restos desprendidos por la mayoría de las especies arbóreas están constituidos de forma absolutamente predominante por hojarasca. Para la encina y melojo, tal predominio no es tan acusado debido a la alta producción de frutos que alcanzan estas especies (ESCUDERO et al., 1984). Por esta razón, conviene estudiar en detalle la distribución de los aportes dẽ nutrientes entre los distintos materiales que se desprenden del arbolado. En la tabla II presentamos los porcentajes con los que cada uno de estos órganos contribuye a la restitución total de cada elemento. Queda claramente puesta de manifiesto la especial importancia de las bellotas en la circulación del potasio, así como en la del fósforo. Esta particular importancia de los frutos se hace aún más evidente cuando se contrasta con la pobre contribución que prestan estos órganos a la circulación total de los demás nutrientes. Particularmente, en la circulación de calcio, sodio, hierro y manganeso el porcentaje de aportación de los frutos es sumamente reducido.

Estas diferencias son importantes porque las bellotas juegan un papel evidentemente distinto al del resto de los materiales desprendidos del árbol. En tanto que la mayoría de estos van a incorporarse directamente al subsistema de los descomponedores en el suelo, las bellotas serán por el contrario ingeridas y asimiladas en gran medida por los animales herbívoros, y los nutrientes incorporados en ellas seguirán así una vía de reciclado completamente distinta.

La vegetación herbácea juega un papel también importante en la dinámica de estos ecosistemas. De hecho, ocupa la mayor parte de su superficie total, como lo atestiguan las cifras de cobertura de arbolado dadas al comienzo de este trabajo. Las extracciones anuales de nutrientes efectuadas por el estrato herbáceo en las parcelas estudiadas se presentan en la tabla III. Resulta llamativo el incremento de extracciónes en las proximidades de los árboles, como respuesta a la fertilización del suelo que efectúan los detritos del arbolado. Pero lo que más interesa destacar es que el potasio juega en el estrato herbáceo un papel consi- 
derablemente más destacado que en el arbóreo, como es habitual en la mayoría de los ecosistemas (DENAEYER-DE SMET, 1971). Para ninguno de los nutrientes, el estrato herbáceo consigue superar la intensidad de circulación promovida por el arbolado en su zona de influencia; sin embargo, la circulación del potasio por unidad de superficie a través del pastizal resulta, en comparación con las restituciones en los detritos arbóreos, bastante más elevada que la de los restantes elementos.

Así pues, del potasio total del ecosistema que anualmente se incorpora en la materia orgánica sintetizada, una parte mucho más significativa que para los restantes elementos lo hace en el material herbáceo en lugar de en los detritos del arbolado. No es preciso recordar que el consumo y asimilación de la hierba por parte de los fitófagos son mucho más probables que los del material detrítico del arbolado. La digestibilidad media de la hierba de diversas comunidades típicas del pastizal de la dehesa (GARCIA et al., 1978) se eleva al $65.10 \%$ de su materia seca total. La de la hojarasca muerta de encina, que es el material detrítico más digerible exceptuados los frutos, sólo supone el $56.29 \%$ (ESCUDERO y col., 1981).

Volvemos, pues, a obtener como resultado el que el potasio aparezca en concentraciones elevadas en un material destinado en buena parte a su ingestión y asimilación por animales herbívoros. Sumadas las contribuciones de los frutos del arbolado y de la hierba, obtenemos como resultado global para todas las parcelas y períodos de estudio el que un $85.49 \%$ del potasio incorporado en la materia orgánica sintetizada en el conjunto de la dehesa se presenta en materiales de relativamente alta digestibilidad y que, por consiguiente, van a ser con mayor probabilidad ingeridos y asimilados por animales herbívoros. Una porción importante del potasio, por consiguiente, no es reciclada por el subsistema de los descomponedores, sino por la cadena trófica herbívorocarnívoro. Como comparación, incluimos a continuación las proporciones de los restantes nutrientes que pasan a formar parte de los materiales vegetales más asimilables:

$\begin{array}{cccccccc}\mathrm{N} & \mathrm{P} & \mathrm{Ca} & \mathrm{Mg} & \mathrm{Na} & \mathrm{Fe} & \mathrm{Cu} & \mathrm{Mn} \\ 66.56 & 77.02 & 39.19 & 59.48 & 77.13 & 56.05 & 53.56 & 37.16\end{array}$

Ninguno de estos elementos presenta una asimilabilidad potencial tan elevada como el potasio. Entre ellos, a su vez, existen considerables diferencias, apareciendo como los menos susceptibles de asimilación para los animales calcio, cobre, hierro y manganeso. Para todos estos elementos, por tanto, la influencia de los herbívoros sobre su circulación es muy reducida. Cabe decir lo contrario de la actividad de los descom- 
ponedores, que será crucial para devolver estos elementos a su forma mineral y asimilable para la vegetación, cerrando así su ciclo.

La incorporación parcial del potasio a la zoocenosis contribuye a acelerar su circulación por el ecosistema. La retención de este nutriente en los tejidos animales es muy pequeña en comparación con la que experimentan otros elementos, como calcio o nitrógeno (PERKINS et al., 1978). La mayor parte del potasio asimilado por un animal vuelve al suelo a través de la orina (WILKINSON y LOWREY, 1973), siendo en consecuencia soluble en agua e inmediatamente disponible para la vegetación. Esta característica contrasta con la relativamente mayor inmovilidad del potasio en los materiales vegetales. Otra consecuencia de la incorporación de nutrientes a los animales es el facilitar las transferencias de un punto a otro del ecosistema, así como su exportación al exterior. Estas transferencias pueden resultar en un aumento de la heterogeneidad espacial incluso por encima de la ya existente a causa de la presencia del arbolado (SWIFT et al., 1978). Pero, más significativamente, el paso de nutrientes a través de sucesivos niveles tróficos tiende a hacer más compleja y dependiente de factores múltiples su circulación por el ecosistema. Estas son, en consecuencia algunas de las características que posee la circulación del potasio, así como la de algunos de los otros nutrientes más limitantes, en comparación con los demás elementos.

\section{B. Transferencias de potasio en el agua de lluvia y de lavado.}

La materia orgánica no constituye el único vehículo de transferencias de nutrientes entre componentes del ecosistema. Considerables proporciones de muchos elementos retornan al suelo antes de la muerte de los tejidos vegetales como consecuencia de su disolución y arrastre por el agua de lluvia. En la tabla IV se presentan las cantidades de potasio que acceden anualmente al suelo en el agua de precipitación atmosféri$\mathrm{ca}$, trascolación y escorrentía. En la tabla $\mathrm{V}$ se reúnen las cifras de adición de potasio y otros nutrientes efectuada por el árbol (es decir, la diferencia entre las cantidades contenidas en la trascolación y escorrentía y las ya existentes en la precipitación atmosférica).

Debido a la extraordinaria variabilidad que presentan tanto las aportaciones de la precipitaciön atmosférica como las transferencias de nutrientes en el agua de lluvia, no consideramos informativo comparar exhaustivamente nuestros datos con los de otros autores. Sin embargo, por lo que respecta a los aportes de potasio en la precipitación atmosférica, nuestros datos parecen indicar una deposición húmeda atmosféri- 
ca en la precipitación ligeramente alta en relación a lo habitual en otros ambientes. Las entradas anuales de potasio en el agua de lluvia según diversos autores (RICHARDSON y MERVA, 1976; DETTORI, 1980; MILLER y MILLER, 1980; TABATABAI et al., 1981, entre otros) varían entre 0.41 y $9.64 \mathrm{~kg} / \mathrm{ha}$. Los valores obtenidos sobre el área de dehesa varían entre 1.96 y $11.52 \mathrm{~kg} / \mathrm{ha}$., por lo que sobrepasan el límite superior dado por los citados autores, aun cuando tampoco faltan otros datos bibliográficos que reportan flujos de potasio en la precipitación atmosférica superiores (FORGEARD et al., 1980, por ejemplo, calculan unas entradas de potasio de $19 \mathrm{~kg}$ ha-1 año-1). Parece que en nuestros datos se hace patente el enriquecimiento del agua de lluvia a partir del potasio del suelo, proceso que suele verificarse en laŝ áreàs más alejadas de la influencia oceánica (MILLER y MILLER, 1980). Frente a otros elementos, también aparece el potasio en posición más destacada de lo que suele ser habitual en otros ambientes:

Todo lo contrario sucede con la adición de potasio al agua de lluvia después de entrar ésta en contacto con el follaje. Los aportes anuales del arbolado de la dehesa oscilan en torno a $7.7 \mathrm{~kg} / \mathrm{ha}$ (tabla V). Estudios de otros autores (AUSSENAC et al., 1972; COLE et al., 1978; FORGEARD et al., 1980; GOSZ et al., 1976; RAPP, 1971, entre otros) dan como aportes medios de potasio por esta vía valores comprendidos entre 10.7 y $84.6 \mathrm{~kg} / \mathrm{ha}$. Por consiguiente, aparece una inversión de los resultados: aun cuando los aportes atmosféricos sobre el área de dehesas no resultan inferiores, sino más bien todo lo contrario, a los de otras regiones, el arrastre de potasio, y también de otros elementos, se hace considerablemente menor.

Esto no impide, sin embargo, que las cantidades de potasio extraídas por el agua de lluvia del arbolado de la dehesa sean considerablemente importantes. Sumadas estas cantidades a la restitución de potasio en los materiales sólidos desprendidos del árbol, se obtienen unos aportes anuales totales comprendidos, según los años y parcelas, entre 5.48 y $9.26 \mathrm{~kg} / \mathrm{ha}$ en las dehesas de encina y entre 6.67 y 8.43 en las de roble. Estas cifras son un promedio calculado para toda la superficie de la dehesa, en tanto que las que hemos dado para el lavado de nutrientes (tabla V) sólo se refieren a la zona directamente cubierta por la copa. Es por esto por lo que estas últimas aparecen comparativamente mayores. De este total, el potasio vinculado al agua de lavado representa del 24 al $36 \%$ para la encina y del 34 al $37 \%$ para el roble. Estas proporciones resultan superiores a las de los restantes elementos con la única excepción del sodio. Como comparación, la circulación del sodio se halla vinculada al lavado en proporciones variables entre 53 y $85 \%$; 
la del nitrógeno, entre 0 y $8 \%$; la del fósforo, entre 11 y $48 \%$; la del calcio, entre 6 y $16 \%$; la del magnesio, finalmente, entre 2.5 y $18 \%$.

\section{Variación estacional de las transferencias de nutrientes.}

Hemos representado la variación estacional del acceso al suelo de tres de los nutrientes más importantes en la figura 1 (aportes en los restos orgánicos desprendidos de los árboles) y en la figura 2 (aportes conjuntos a través de los restos orgánicos y del lavado por el agua de lluvia).

La caída de los restos orgánicos de las encinas se verifica mayoritariamente en dos épocas del año: finales de primavera (caída de hojas, ramillas y amentos masculinos) y a mediados de otoño (desprendimiento de glandes con sus cúpulas). Por el contrario, el roble, como especie caducifolia, sufre una abscisión foliar mayoritaria hacia el mes de noviembre, a lo que se une la caída en la misma época de los frutos. Sin embargo, también a finales de la primavera se verifica la caída de las inflorescencias junto con una defoliación adicional provocada por la actividad de orugas defoliadoras. Estos materiales tienen una elevada riqueza en elementos nutritivos; por lo que su aportación de nutrientes resulta bastante más importante de lo que cabría esperar por la pequeña masa de materia orgánica que suponen.

Estas pautas de variación en la aportación de detritos se reflejan claramente en la restitución de nutrientes (fig. 1). La restitución de los tres nutrientes presenta una neta bimodalidad anual en la encina. Sin embargo, las diferencias en composición química entre los glandes, por un lado, y hojas e inflorescencias, por otro, hacen que esta pauta de variación difiera en cierta medida de unos elementos a otros. El potasio es el nutriente que presenta aportes otoñales más destacados, a causa de su elevada concentración en las bellotas. En el roble (parcela de Cilloruelo) también los aportes otoñales de este elemento se ven destacados por la misma razón. Por el contrario, fósforo y sobre todo nitrógeno presentan restituciones primaverales muy elevadas, porque estos nutrientes son abundantes en las inflorescencias masculinas y en las hojas verdes desprendidas de los robles en esta época.

La intervención del lavado destruye en gran parte estas regularidades. Este proceso es, en realidad, demasiado dependiente de los eventos meteorológicos y de otros factores más o menos aleatorios como para poder ajustarse a alguna pauta de variación estacional. Su efecto sobre la regularidad de la aportación total de nutrientes depende, naturalmente, de la importancia de la contribución relativa del lavado a la cesión total 


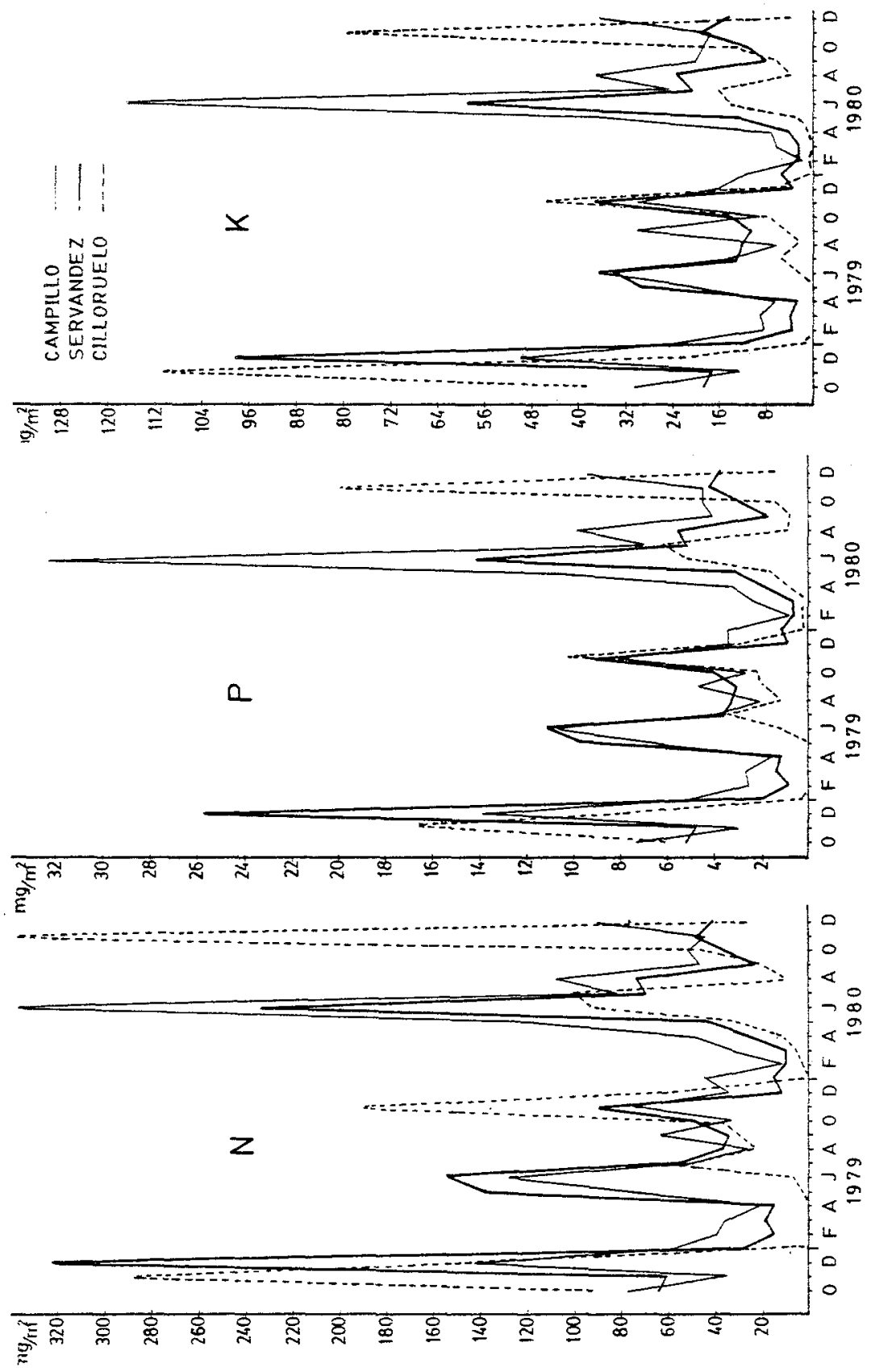

Figura 1.-Variación estacional de los aportes detríticos de nitrógeno, fósforo y potasio (en $\mathrm{mg} / \mathrm{m}^{2}$ ). 

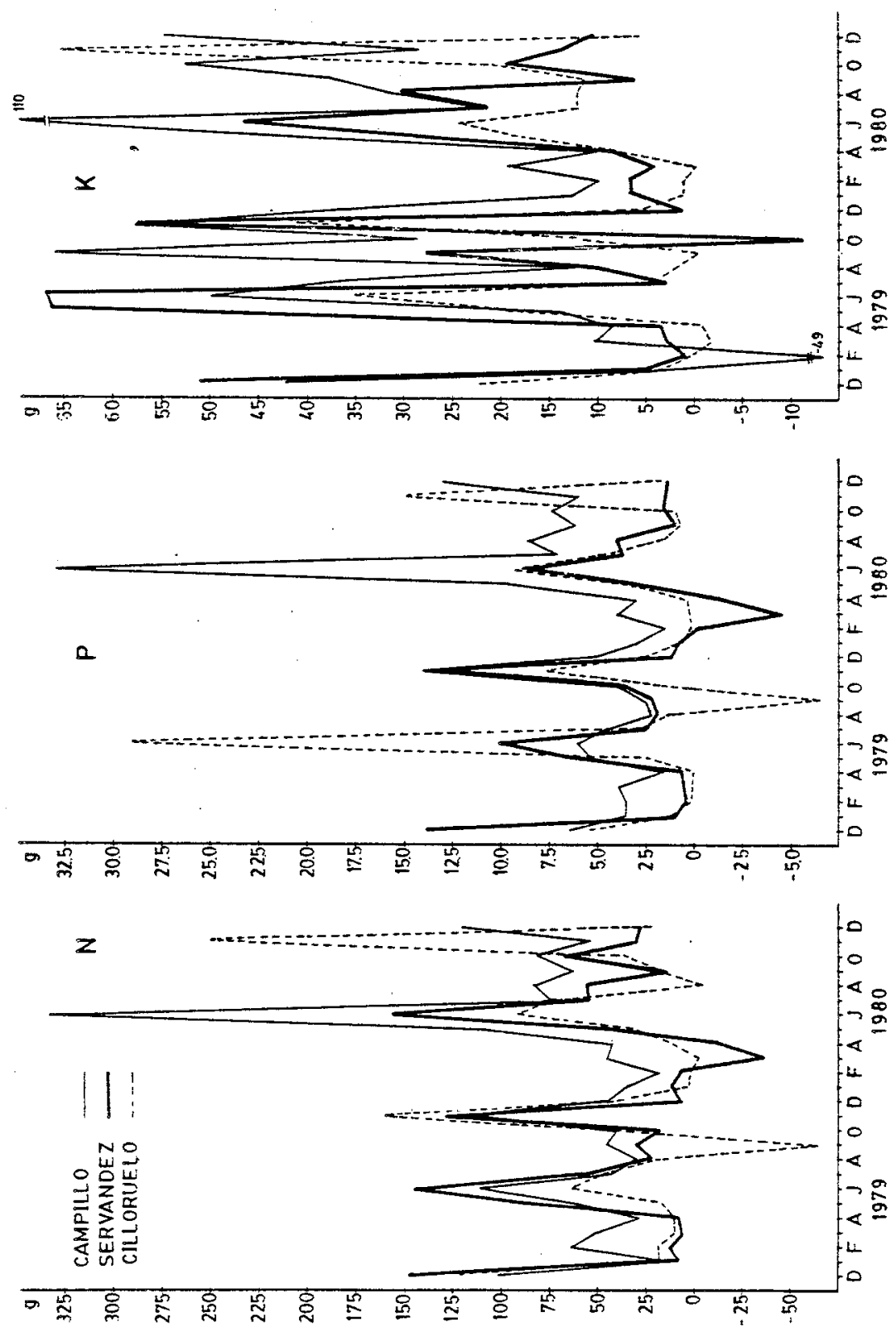

Figura 2.-Variación estacional de las cantidades totales de nitrógeno, fósforo y potasio puestas en circulación a través de los detritos y del lavado. 
de elementos de la copa al suelo. Aquí volvemos a encontrar una diferencia sustancial entre el potasio y otros macronutrientes esenciales. La figura 2 muestra cómo los flujos de nitrógeno y fósforo siguen presentando de manera claramente reconocible el patrón de variación característico de la deposición de restos orgánicos. Por el contrario, el potasio no manifiesta casi en absoluto pautas estacionales a causa de la importancia del lavado en su circulación total. La consecuencia más importante de esta irregularización es el posible trastorno causado a la sincronización de la actividad de las poblaciones vegetales y edáficas para el mejor uso de los recursos puestos a su disposición y la minimización de las pérdidas de los mismos. Este efecto negativo incide, por tanto, en mayor medida sobre la economía del potasio en el ecosistema que sobre la de ningún otro de los macronutrientes esenciales.

\section{Modificación de la fertilidad del suelo inducida por el arbolado.}

La modificación de la fertilidad del suelo en la proximidad del arbolado constituye una representación muy adecuada del efecto global de los aportes de materiales procedentes de los árboles. En la tabla I hemos presentado las propiedades fisicoquímicas de los suelos de las parcelas estudiadas en las zonas libres de los aportes del arbolado. En la tabla VI incluimos ahora los valores de los índices que se obtienen dividiendo el valor de cada parámetro en la zona situada debajo de las copas entre el valor alcanzado por el mismo parámetro en las áreas de pastizal abierto. Como siempre, estos valores son los promedios obtenidos a partir de un cierto número de muestras tomadas bajo distintos árboles. La denominación «tronco» se refiere a muestras tomadas junto a la base del fuste de los árboles para estimar el efecto del agua de escorrentía cortical en esa zona particular.

En la mayoría de los casos, el suelo se enriquece (índices superiores a 1) en las proximidades del arbolado. También suele observarse un incremento del $\mathrm{pH}$, así como de la relación $\mathrm{C} / \mathrm{N}$. Todos estos efectos son más importantes en la proximidad del tronco como consecuencia de los fuertes contenidos de elementos minerales del agua de escorrentía. Los contenidos de potasio asimilable figuran entre los más afectados por la presencia del arbolado. En particular, en la base del tronco las adiciones de este elemento en el agua de escorrentía se patentizan en elevados índices de enriquecimiento. Sin embargo, también se pone de manifiesto en estos resultados la peculiaridad del potasio frente a los restantes elementos: mientras que para todos estos últimos el enriquecimiento por efecto del arbolado tiene ante todo lugar en la superficie del suelo y muy poco en profundidad (como excepción se presenta 
la parcela de roble, a causa seguramente de una gran carga ganadera sobre ella), con el potasio sucede todo lo contrario. La solubilidad del potasio favorece evidentemente su arrastre hacia los niveles inferiores del perfil y el enriquecimiento de éstos. La consecuencia es una modificación por el arbolado de la distribución del potasío a lo largo del perfil respecto de lo normal en estos suelos. En tanto que en ausencia de aportes arbóreos los niveles superficiales de potasio suelen ser mucho más elevados que en profundidad (tabla I), la presencia del arbolado determina unos niveles de potasio más similares a distintas profundidades.

\section{CONCLUSIONES}

Todos los datos que hemos aportado coinciden en asignar al ciclo del potasio unas características muy especiales frente al de los restantes elementos. En primer lugar, ha de destacarse su mayor complejidad. Una buena parte de la cantidad total de potasio incorporada anualmente en la materia vegetal del ecosistema no sigue las rutas de circulación más habituales a través del subsistema de los descomponedores, sino que por el contrario pasa a la cadena trófica de los herbívoros.

El ciclo del potasio es también más complejo y, a la vez, más difícil de cuantificar a causa de la gran movilidad del elemento. Gran parte de sus transferencias tienen lugar a través del agua de lluvia, y sus aportes desde las copas sớn bastante erráticos en el tiempo, a diferencia de la clara estacionalidad de la restitución de los demás macronutrientes esenciales. Todo esto hace bastante probable la participación del potasio en varios ciclos anuales: nada impide el que ciertas cantidades de potasio restituidas al suelo en épocas favorables por disolución en el agua de lavado sean tomadas nuevamente por las raíces en razón de su inmediata disponibilidad. La probabilidad de participar en varios ciclos anuales es mucho menor para los restantes elementos; su restitución al suelo tiene lugar preferentemente en épocas poco favorables para la actividad vegetal, aparte de precisar períodos más o menos largos para su liberación de la materia orgánica y consiguiente incremento de su disponibilidad para la vegetación.

La complejidad del ciclo del potasio debería contribuir a incrementar su estabilidad. Sin embargo, también es cierto que su solubilidad y la irregularidad de su acceso al suelo pueden favorecer las pérdidas por percolación. Nosotros no hemos medido este flujo, aunque los datos edảficos (tabla VI) apoyan la suposición de un intenso movimiento del potasio en el suelo. 
La fuerte intervención de los animales herbívoros en la circulación del potasio hace, finalmente, más importante la intervención humana en la distribución espacial de este elemento. Un manejo adecuado del ganado podría hacer más favorable esta distribución, aunque quizá sea lo más frecuente el que suceda todo lo contrario. 


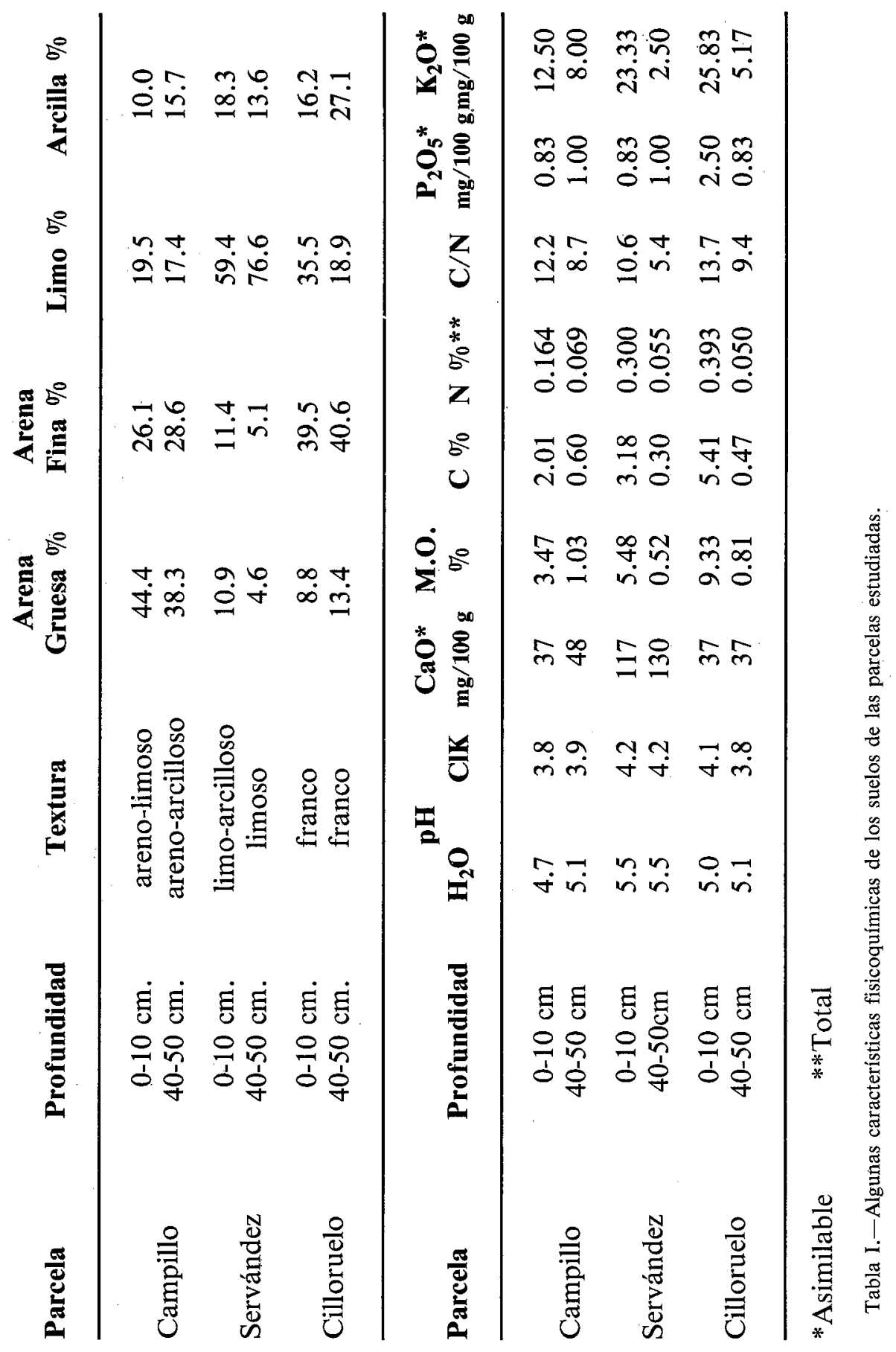




\begin{tabular}{lrrrrrr}
\hline $\mathrm{K}$ & 44.86 & 11.98 & 11.56 & 18.56 & 7.60 & 5.46 \\
$\mathrm{~N}$ & 46.20 & 10.77 & 6.05 & 16.79 & 13.41 & 6.79 \\
$\mathrm{P}$ & 45.51 & 11.77 & 5.59 & 21.09 & 10.01 & 6.04 \\
$\mathrm{Ca}$ & 57.76 & 22.12 & 3.30 & 2.83 & 6.60 & 7.41 \\
$\mathrm{Mg}$ & 54.24 & 14.13 & 6.58 & 11.73 & 8.07 & 5.27 \\
$\mathrm{Na}$ & 68.65 & 13.98 & 5.55 & 2.30 & 5.20 & 4.34 \\
$\mathrm{Fe}$ & 54.36 & 11.82 & 3.03 & 3.06 & 11.54 & 16.19 \\
$\mathrm{Cu}$ & 34.54 & 16.75 & 7.74 & 17.06 & 14.92 & 8.51 \\
$\mathrm{Mn}$ & 74.72 & 7.30 & 3.37 & 3.35 & 6.77 & 4.51 \\
\hline
\end{tabular}

otros roble hojas ramas cúpulas frutos amentos restos

\begin{tabular}{lrrrrrr}
\hline $\mathrm{K}$ & 49.11 & 16.60 & 2.62 & 26.52 & 2.43 & 2.72 \\
$\mathrm{~N}$ & 58.19 & 12.16 & 1.72 & 13.97 & 8.58 & 5.39 \\
$\mathrm{P}$ & 52.60 & 15.70 & 1.55 & 17.00 & 7.49 & 5.65 \\
$\mathrm{Ca}$ & 70.09 & 22.24 & 1.09 & 1.07 & 2.71 & 2.81 \\
$\mathrm{Mg}$ & 70.57 & 15.70 & 2.07 & 4.71 & 4.27 & 2.69 \\
$\mathrm{Na}$ & 68.51 & 27.76 & 0.42 & 0.12 & 2.11 & 1.08 \\
$\mathrm{Fe}$ & 77.02 & 8.73 & 0.94 & 1.89 & 6.40 & 5.02 \\
$\mathrm{Cu}$ & 50.82 & 20.74 & 3.84 & 12.85 & 6.65 & 5.09 \\
$\mathrm{Mn}$ & 81.61 & 10.65 & 1.30 & 1.04 & 3.38 & 2.02 \\
\hline
\end{tabular}

Tabla II.-Contribución (porcentaje) de los diversos materiales al retorno anual de nutrientes por vía detrítica. 


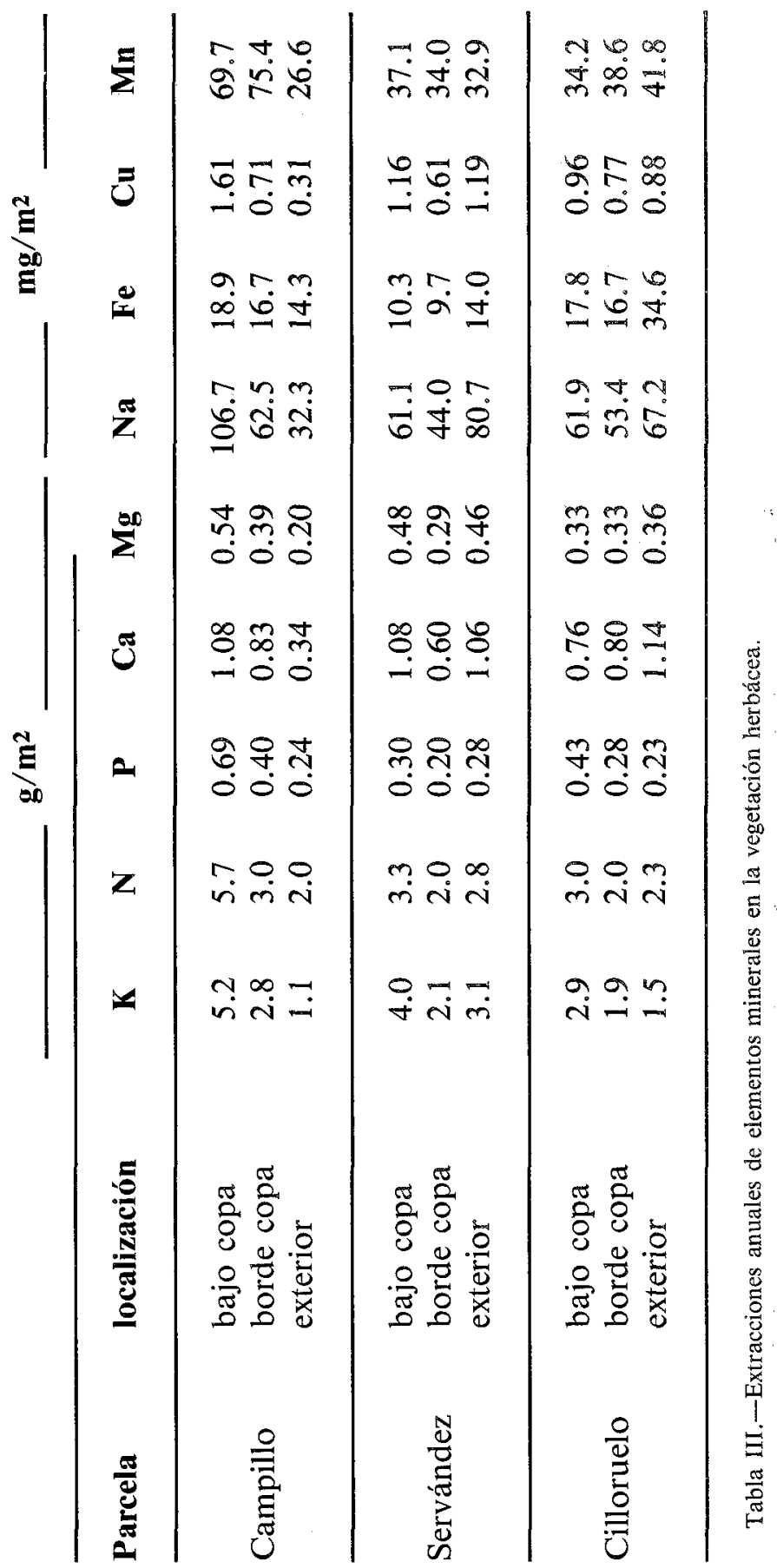


Campillo

$\begin{array}{rrrrr}158.3 & 1474.3 & 1829.8 & 947.7 & 674.1 \\ 16.6 & 1582.6 & 2100.7 & 755.5 & 698.0 \\ 37.9 & 1316.6 & 1743.2 & 433.4 & 446.5\end{array}$

Tabla IV.-Entradas anuales de potasio al suelo $\left(\mathrm{mg} / \mathrm{m}^{2}\right)$ a través de la precipitación atmosférica, trascolación y escorrentía.

\begin{tabular}{lccccccccc} 
Especie & K & N & P & Ca & Mg & Na & Fe & Cu & Mn \\
\hline encina & 773.5 & 125.3 & 38.4 & 837.5 & 95.6 & 126.2 & -3.47 & 0.88 & 37.0 \\
roble & 765.2 & 168.9 & 175.2 & 567.5 & 220.2 & 108.8 & 6.03 & 1.47 & 17.0
\end{tabular}

Tabla V.-Adiciones anuales de nutrientes $\left(\mathrm{en} \mathrm{mg} / \mathrm{m}^{2}\right.$ ) al agua de lavado. 


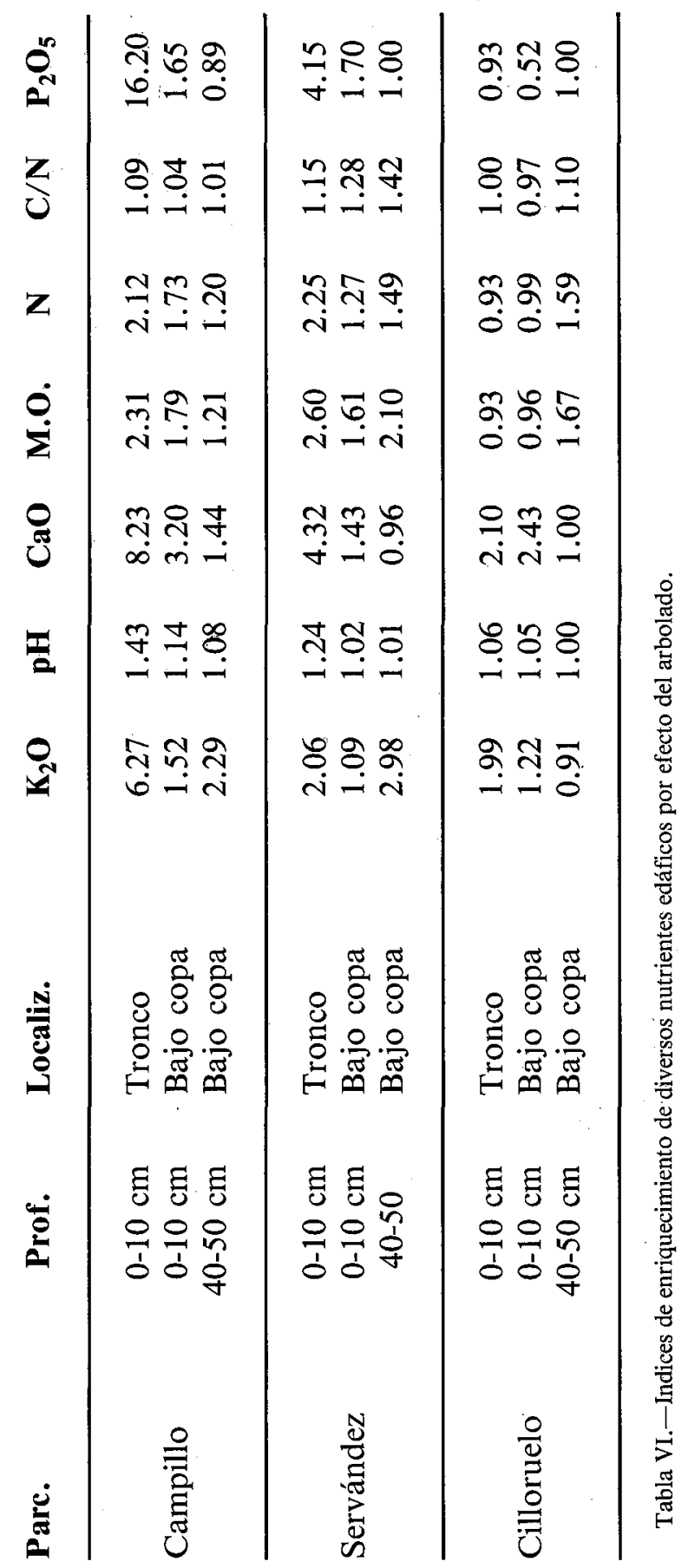


AUSSENAC, G.; BONNEAU, M.; et LE TACON, F. (1972). Restitucion des éléments minéraux au sol par l'intermédiaire de la litière et des précipitations dans quatre peuplements forestiers d l'est de la France. Oecol. Plant. 7, 1-21.

BARES, R.H. and WALI, M.K. (1979). Chemical relations and litter production of Picea maria$n a$ and Larix laricina stands on an alkaline peatland in northern Minnesota. Vegetatio 40, 79-94.

COLE, D.W.; GESSEL, S.P. and TURNER, J. (1978). Comparative mineral cycling in red alder and Douglas-firm. Tech. Rep., USDA For. Serv., Pac. Northwest For, Range Exp. Stn. 7, 327-336.

DENAEYER-DE SMET, S. (1971). Teneurs en éléments biogènes des tapis végétaux dans les forêts caducifoliées d'Europe. In: Productivité des écosystémes forestiers. UNESCO, 515-525.

DETTORI, B. (1979). First observations on the saline content of the precipitations in some stations in Sardinia (Italy). Studi Sassaresi 27, 1-18.

DUVIGNEAUD, P. (1968). Recherches sur l'écosystème forêt. Série D: la chênaie-frênaie à coudrier du bọis de Wève. Aperçu sur la biomasse, la productivité et le cycle des êléments biogènes. Bull. Soc. roy. Bot. Belg. 101, 111-127.

ESCUDERO, A.; GARCIA, B.; LUIS, E. y GOMEZ, J.M. (1980). Materiales aportados al suelo por la encina en la zona de dehesas de la provincia de Salamanca. III. Porcentajes de fracciones orgánicas y de materia digestible. Studia Oecologica 2, 241-266.

ESCUDERO, A.; GARCIA, B.; GOMEZ, J.M.; and LUIS, E. (1984). The nutrient cycling in Quercus rotundifolia and Quercus pyrenaica ecosystems («dehesas») of Spain. Oecol. Plant. (in press).

FORGEARD, F.; GLOAGUEN, J.C.; et TOUFFET, J. (1980). Interception des précipitations et apport au sol d'éléments minéraux par les eaux de pluie et les pluviolessivats dans une hêtraie atlantique et dans quelques peuplements résineux en Bretagne. Ann. Sci. Forest. 37, 53-71.

GARCIA RODRIGUEZ, A. (1964). Los suelos de la provincia de Salamanca. Publicaciones del I.O.A.T.O., Salamanca, $145 \mathrm{pp}$.

GARCIA, B; ESCUDERO, A.; PUERTO, A. and GOMEZ, J.M. (1978). A study of the heterogeneity of herbaceous communities by means of their floristic composition and feeding value. 7th General Meeting, Europ. Grassld. Fed., Gent-Belgium, 8, 69-83.

GOMEZ, J.M.; LUIS, E.; y ESCUDERO, A. (1980). Materiales aportados al suelo por la encina en la zona de dehesas salmantina. 1. Sustancia seca. Studia Oecologica 1, 2, 181-212.

GOSZ, J.R.; LIKENS, G.E. and BORMANN, F.H. (1976). Organic matter and nutrient dynamics of the forest and forest floor in the Hubbard Brook forest. Oecologia 22, 305-320.

MEDWECKA-KORNAS, A. (1971). Plant litter. In: Methods of study in quantitative soil ecology. IBP, Blackwell Scientific Publ., Oxford and Edinburgh, 24-33.

MILLER, H.G. and MILLER, J.D. (1980). Collection and retention of atmospheric pollutants by vegetation. Proc. Int. Conf. Ecol. Impact Acid Precipitation, Norway, SNSF Project.

PERKINS, D.F.; JONES, V.; MILLAR, R.O. and NEEP, P. (1978). Primary production, mineral nutrients and litter decomposition in the grassland ecosystem. In: Production ecology of British moors and montane grasslands, O.W. Heal and D.F. Perkins (eds.), SpringerVerlag, Berlin. 
RAPP, M. (1967). Production de litière et apport au sol d'éléments minéraux et d'azote dans un bois de pins d'Alep ((Pinus halepensis Mill.). Oecol. Plant. 2, 325-338.

RAPP, M. (1969). Production de litière et apport au sol d'éléments minéraux dans deux écosystèmes méditerranéens: la forêt de Quercus ilex L. et la garrigue de Quercus coccifera L. Oecol. Plant. 4, 377-410.

RAPP, M. (1971). Cycle de la matière organique et des éléments minéraux dans quelques écosystèmes méditerranéens. Editions du Centre National de la Recherche Scientifique. Paris, $253 \mathrm{pp}$.

RICHARDSON, C.J. and MERVA, G.E. (1976). The Chemical composition of atmospheric precipitation from selected stations in Michigan. Water, Air and Soil Pollution 6, 385-393.

SWIFT, M.J.; HEAL, O.W. and ANDERSON, J.M. (1979). Decomposition in terrestrial ecosystems. Studies in Ecology vol. 5, Blackwell Scientific Publ., Oxford, 372 pp.

TABATABAI, M.A.; BURWELL, R.E.; ELLIS, B.G.; KEENEY, D.R.; LOGAN, T.J.; NELSON, D.W.; OLSON, R.A.; RANDALL, G.W.; TIMMONS, D.R.; VERRY, E.S. and WHITE, E.M. (1981). Nutrient concentrations and accumulations in precipitation over the North Central Region. North Centrăl Regional Research Publ. No. 282, 111-141.

WILKINSON, S.R. and LOWREY, R.W. (1973). Cycling of mineral nutrients in pasture ecosystems. In. Chemistry and Biochemistry of Herbage, G.W. Butler and R.W. Bailey (eds.) Academic Press, London, 247-315. 